University of Wollongong

Research Online

Australian Institute for Innovative Materials -

Papers

Australian Institute for Innovative Materials

$1-1-2020$

$\mathrm{BiOBr}$ nanoflakes with strong Kerr nonlinearity towards hybrid integrated photonic devices

Linnan Jia

Dandan Cui

Jiayang Wu

Haifeng Feng

University of Wollongong, haifeng@uow.edu.au

Yunyi Yang

See next page for additional authors

Follow this and additional works at: https://ro.uow.edu.au/aiimpapers

Part of the Engineering Commons, and the Physical Sciences and Mathematics Commons

Research Online is the open access institutional repository for the University of Wollongong. For further information contact the UOW Library: research-pubs@uow.edu.au 


\title{
$\mathrm{BiOBr}$ nanoflakes with strong Kerr nonlinearity towards hybrid integrated photonic devices
}

\author{
Abstract \\ (C) 2020 SPIE. As a new group of advanced 2D layered materials, bismuth oxyhalides, i.e., $\mathrm{BiOX}(\mathrm{X}=\mathrm{Cl}, \mathrm{Br}$, \\ I), have recently become of great interest. In this work, we characterize the third-order optical \\ nonlinearities of $\mathrm{BiOBr}$, an important member of the BiOX family. The nonlinear absorption and Kerr \\ nonlinearity of $\mathrm{BiOBr}$ nanoflakes at both $800 \mathrm{~nm}$ and $1550 \mathrm{~nm}$ are characterized via the Z-Scan technique. \\ Experimental results show that $\mathrm{BiOBr}$ nanoflakes exhibit a large nonlinear absorption coefficient $\beta \sim 10-7$ \\ $\mathrm{m} / \mathrm{W}$ as well as a large Kerr coefficient $\mathrm{n} 2 \sim 10-14 \mathrm{~m} 2 / \mathrm{W}$. We also note that the $\mathrm{n} 2$ of $\mathrm{BiOBr}$ reverses sign \\ from negative to positive as the wavelength is changed from $800 \mathrm{~nm}$ to $1550 \mathrm{~nm}$. We further characterize \\ the thickness-dependent nonlinear optical properties of $\mathrm{BiOBr}$ nanoflakes, finding that the magnitudes of \\ $\beta$ and $n 2$ increase with decreasing thickness of the $\mathrm{BiOBr}$ nanoflakes. Finally, we integrate $\mathrm{BiOBr}$ \\ nanoflakes into silicon integrated waveguides and measure their insertion loss, with the extracted \\ waveguide propagation loss showing good agreement with mode simulations based on ellipsometry \\ measurements. These results confirm the strong potential of $\mathrm{BiOBr}$ as a promising nonlinear optical \\ material for high-performance hybrid integrated photonic devices. \\ Disciplines \\ Engineering | Physical Sciences and Mathematics

\section{Publication Details} \\ Jia, L., Cui, D., Wu, J., Feng, H., Yang, Y., Yang, T., Qu, Y., Du, Y., Hao, W., Jia, B. \& Moss, D. (2020). BiOBr \\ nanoflakes with strong Kerr nonlinearity towards hybrid integrated photonic devices. Proceedings of SPIE \\ - The International Society for Optical Engineering, 11282
}

\section{Authors}

Linnan Jia, Dandan Cui, Jiayang Wu, Haifeng Feng, Yunyi Yang, Tieshan Yang, Yang Qu, Yi Du, Weichang Hao, Baohua Jia, and David Moss 


\section{$\mathrm{BiOBr}$ nanoflakes with strong Kerr nonlinearity towards hybrid integrated photonic devices}

Jia, Linnan, Cui, Dandan, Wu, Jiayang, Feng, Haifeng, Yang, Yunyi, et al.

Linnan Jia, Dandan Cui, Jiayang Wu, Haifeng Feng, Yunyi Yang, Tieshan Yang, Yang Qu, Yi Du, Weichang Hao, Baohua Jia, David J. Moss, "BiOBr nanoflakes with strong Kerr nonlinearity towards hybrid integrated photonic devices," Proc. SPIE 11282, 2D Photonic Materials and Devices III, 112820Q (18 February 2020); doi: 10.1117/12.2546243

SPIE. Event: SPIE OPTO, 2020, San Francisco, California, United States 


\title{
BiOBr nanoflakes with strong Kerr nonlinearity towards hybrid integrated photonic devices
}

\author{
Linnan Jia ${ }^{\mathrm{a}}$, Dandan Cui ${ }^{\mathrm{b}}$, Jiayang $\mathrm{Wu}^{\mathrm{a}}$, Haifeng Feng ${ }^{\mathrm{c}}$, Yunyi Yang ${ }^{\mathrm{d}}$, \\ Tieshan Yang ${ }^{\mathrm{d}}$, Yang $\mathrm{Qu}^{\mathrm{a}}$, Yi Duc ${ }^{\mathrm{c}}$, Weichang Hao ${ }^{\mathrm{b}}$, Baohua Jia ${ }^{\mathrm{a}, \mathrm{d}}$, and David J. Moss ${ }^{\mathrm{a} *}$ \\ ${ }^{a}$ Centre for Micro-Photonics, Swinburne University of Technology, Hawthorn, VIC 3122, Australia; \\ ${ }^{b}$ School of Physics, and BUAA-UOW Joint Research Centre, Beihang University, Beijing 100191, China; \\ 'Institute for Superconducting and Electronic Materials, and UOW-BUAA Joint Research Centre, University of \\ Wollongong, Wollongong, NSW 2500, Australia; \\ ${ }^{\mathrm{d} C}$ Centre for Translational Atomaterials, Swinburne University of Technology, Hawthorn, VIC 3122, Australia \\ * Electronic mail: $\underline{\text { dmoss@ @swin.edu.au }}$
}

\begin{abstract}
As a new group of advanced 2D layered materials, bismuth oxyhalides, i.e., $\mathrm{BiOX}(\mathrm{X}=\mathrm{Cl}, \mathrm{Br}$, I), have recently become of great interest. In this work, we characterize the third-order optical nonlinearities of $\mathrm{BiOBr}$, an important member of the BiOX family. The nonlinear absorption and Kerr nonlinearity of BiOBr nanoflakes at both $800 \mathrm{~nm}$ and $1550 \mathrm{~nm}$ are characterized via the Z-Scan technique. Experimental results show that $\mathrm{BiOBr}$ nanoflakes exhibit a large nonlinear absorption coefficient $\beta \sim 10^{-7} \mathrm{~m} / \mathrm{W}$ as well as a large Kerr coefficient $n_{2} \sim 10^{-14} \mathrm{~m}^{2} / \mathrm{W}$. We also note that the $n_{2}$ of $\mathrm{BiOBr}$ reverses sign from negative to positive as the wavelength is changed from $800 \mathrm{~nm}$ to $1550 \mathrm{~nm}$. We further characterize the thickness-dependent nonlinear optical properties of $\mathrm{BiOBr}$ nanoflakes, finding that the magnitudes of $\beta$ and $n_{2}$ increase with decreasing thickness of the $\mathrm{BiOBr}$ nanoflakes. Finally, we integrate $\mathrm{BiOBr}$ nanoflakes into silicon integrated waveguides and measure their insertion loss, with the extracted waveguide propagation loss showing good agreement with mode simulations based on ellipsometry measurements. These results confirm the strong potential of $\mathrm{BiOBr}$ as a promising nonlinear optical material for high-performance hybrid integrated photonic devices.
\end{abstract}

Keywords: 2D materials, $\mathrm{BiOBr}$ nanoflake, Kerr nonlinearity, integrated photonics

\section{INTRODUCTION}

Featuring broad operation bandwidths, low power consumption, and potentially reduced cost, all-optical signal processing based on nonlinear photonic devices has provided a competitive solution to realize ultrafast signal processing.[1-7] As the key building blocks for implementing nonlinear photonic devices, advanced optical materials with superior nonlinear properties have been widely investigated. Recently, two-dimensional (2D) layered materials such as graphene[8, 9], graphene oxide (GO) [10-14], transition metal dichalcogenides (TMDCs) [15-17], and black phosphorus (BP) [18-20] have attracted significant interest with their remarkable optical properties, such as ultrahigh Kerr optical nonlinearities, strong nonlinear absorption, significant material anisotropy, and layer-dependent material properties. These $2 \mathrm{D}$ materials have already enabled various new photonic devices which are fundamentally different from those based on traditional bulk materials [21-23].

In addition to those typical 2D materials, Bismuth oxyhalides, i.e., $\mathrm{BiOX}(\mathrm{X}=\mathrm{Cl}, \mathrm{Br}$, I), have been explored as a new group of advanced 2D layered materials [24-26]. BiOX have an open-layer crystal structure which is built by interlacing $\left[\mathrm{Bi}_{2} \mathrm{O}_{2}\right]$ slabs with double halogen slabs. This unique structure enables self-built internal static electric fields in BiOX, which lead to an effective separation of photoinduced charge carriers, thus enabling BiOX with superior photocatalytic behavior [26-28] and excellent nonlinear optical performance [29, 30]. The strong nonlinear optical properties of $\mathrm{BiOCl}$, in terms of both nonlinear absorption and Kerr nonlinearity, have been demonstrated at $515 \mathrm{~nm}[29]$ and $800 \mathrm{~nm}[30]$.

In this work, we investigate the nonlinear optical properties of BiOBr nanoflakes. The nonlinear absorption and Kerr nonlinearity of BiOBr nanoflakes at both $800 \mathrm{~nm}$ and $1550 \mathrm{~nm}$ are characterized via the Z-Scan technique [31]. We demonstrate $\mathrm{BiOBr}$ nanoflakes have strong nonlinear absorption and high Kerr nonlinearity at both wavelengths, 
with a large nonlinear absorption coefficient $\beta$ of $\sim 10^{-7} \mathrm{~m} / \mathrm{W}$ as well as a large Kerr coefficient $n_{2} \sim 10^{-14} \mathrm{~m}^{2} / \mathrm{W}$. A strong dispersion of Kerr coefficient $n_{2}$ of $\mathrm{BiOBr}$ is also observed in our experiment, where $n_{2}$ reverses sign from negative to positive as the wavelength is changed from $800 \mathrm{~nm}$ to $1550 \mathrm{~nm}$. In addition, thickness dependent nonlinear response of the $\mathrm{BiOBr}$ nanoflakes are verified, with $\beta$ and $n_{2}$ increasing for very thin flake thicknesses. We also integrate the $\mathrm{BiOBr}$ nanoflakes onto silicon integrated waveguides and measure the insertion loss of the hybrid integrated devices, with the extracted waveguide propagation loss showing good agreement with mode simulations based on ellipsometry measurements. Our results verify the strong potential of $\mathrm{BiOBr}$ as an advanced optical material for high-performance nonlinear photonic devices.

\section{MATERIAL PREPARATION AND CHARACTERIZATION}

Fig. 1(a) illustrates the atomic structure of $\mathrm{BiOBr}$ crystals, where $\left[\mathrm{Bi}_{2} \mathrm{O}_{2}\right]^{2+}$ slabs are interleaved by double $\mathrm{Br}$ atoms to form a layered structure $[24,26]$. The thickness profiles of the prepared $\mathrm{BiOBr}$ nanoflakes were characterized by atomic force microscopy (AFM), as shown in Fig. 1(b). The measured thicknesses of the samples in (i) - (iv) are $~ 30$ $\mathrm{nm}, \sim 75 \mathrm{~nm}, \sim 110 \mathrm{~nm}$, and $\sim 140 \mathrm{~nm}$, respectively.

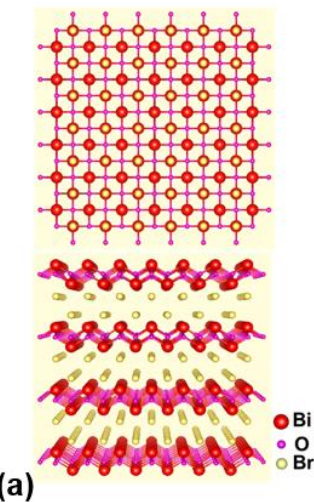

(b)
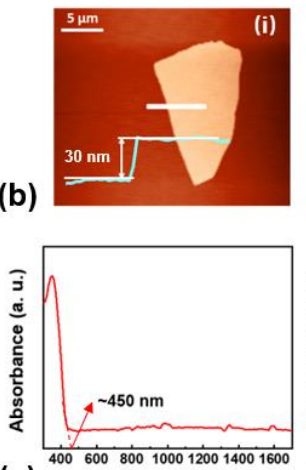

(c)
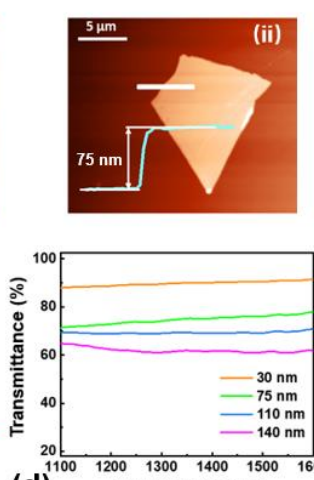

(d)
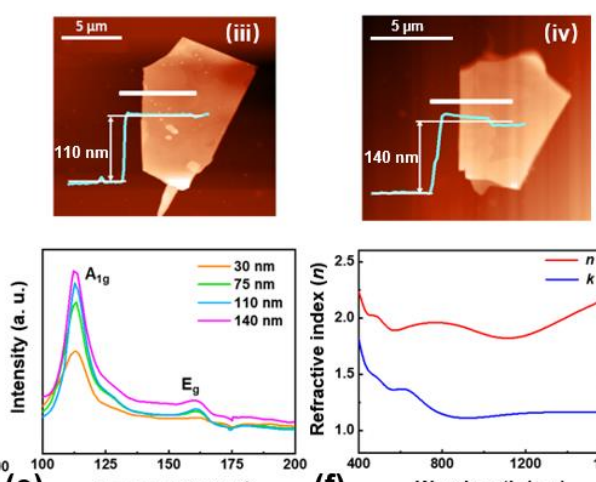

$(\mathrm{e})$

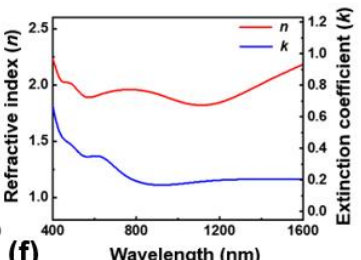

(f) Wavelength (nm)

Fig. 1. (a) Schematic atomic structure of BiOBr. (b) AFM images and height profiles of exfoliated BiOBr nanoflakes with various thicknesses: (i) $\sim 30 \mathrm{~nm}$, (ii) $\sim 75 \mathrm{~nm}$, (iii) $\sim 110 \mathrm{~nm}$, (iv) $\sim 140 \mathrm{~nm}$. (c) UV-vis absorption spectrum of BiOBr. (d) Measured linear transmittance spectra of $\mathrm{BiOBr}$ nanoflakes with different thicknesses. (e) Raman spectra of BiOBr nanoflakes with different thicknesses. (f) Measured refractive index $(n)$ and extinction coefficient $(k)$ of $\mathrm{BiOBr}$.

Fig. 1(c) depicts the linear absorption of $\mathrm{BiOBr}$ from $300 \mathrm{~nm}$ to $1700 \mathrm{~nm}$ measured by ultraviolet-visible (UV-vis) spectrometry. A strong linear absorption edge near $\sim 450 \mathrm{~nm}$ is observed, which corresponds to a photon energy of $2.76 \mathrm{eV}$, in agreement with the reported bandgap of $\mathrm{BiOBr}[28]$. We also measured the transmittance spectra of $\mathrm{BiOBr}$ nanoflakes with different thicknesses, as shown in Fig. 1(d). High transmittance (> 60\%) for wavelengths from 1100 $\mathrm{nm}$ to $1600 \mathrm{~nm}$ is observed for all samples, with a transmittance reaching $90 \%$ for the $30-\mathrm{nm}$-thick BiOBr nanoflakes. To characterize the quality of prepared $\mathrm{BiOBr}$ samples, Raman spectroscopic measurements were conducted with a pump laser wavelength of $\sim 532 \mathrm{~nm}$. As shown in Fig. 1 (e), two phonon modes of $A_{1 g}\left(\sim 113.2 \mathrm{~cm}^{-1}\right)$ and $\mathrm{E}_{\mathrm{g}}(\sim 160.4$ $\mathrm{cm}^{-1}$ ) are observed for all samples, which is consistent with previous reports [32, 33]. Fig. 1(f) shows the in-plane refractive index $(n)$ as well as extinction coefficient $(k)$ of $\mathrm{BiOBr}$ measured by spectral ellipsometry [11, 34]. The sample thickness is $\sim 1 \mu \mathrm{m}$. The measured $n$ and $k$ in telecommunications band are $\sim 2.2$ and $\sim 0.2$, respectively.

\section{Z-SCAN MEASUREMENT}

We characterize the nonlinear absorption and refraction of the prepared $\mathrm{BiOBr}$ samples via Z-scan techniques. [20, $31,35]$ The experimental setup is illustrated in Fig. 2. Femtosecond pulsed lasers, with center wavelengths at $\sim 800$ $\mathrm{nm}$ and $\sim 1550 \mathrm{~nm}$, were used to excite the samples. The repetition rate and pulse duration were $\sim 80 \mathrm{MHz}$ and $\sim 140$ fs, respectively. The incident laser beam was focused by an objective lens (10x, $0.25 \mathrm{NA})$ to achieve a low beam waist with a focused spot size much smaller than the sample size, at $\sim 1.6 \mu \mathrm{m}$ and $\sim 3.1 \mu \mathrm{m}$ for the $800-\mathrm{nm}$ and $1550-\mathrm{nm}$ pulsed lasers, respectively. 


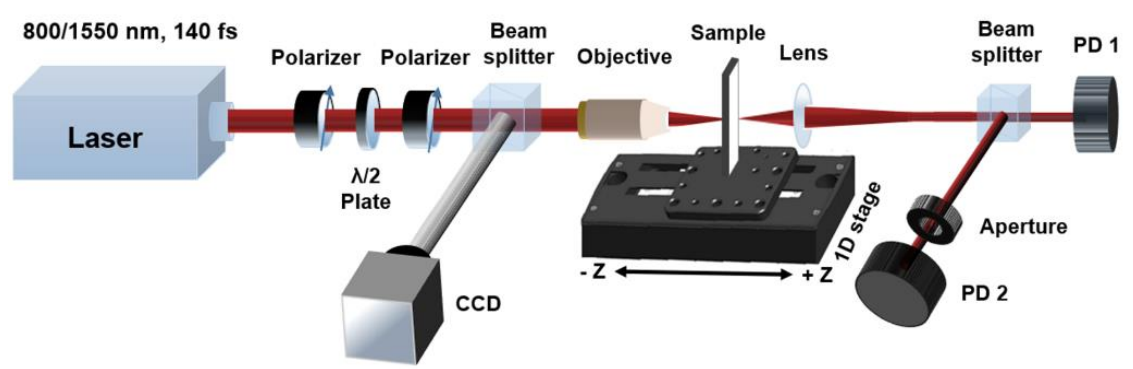

Fig. 2. Schemes illustration of Z-scan experimental setup. PD: power detector.

Fig. 3(a) and (b) depict the Z-scan results of a BiOBr nanoflake $(\sim 140 \mathrm{~nm})$ at $800 \mathrm{~nm}$ and $1550 \mathrm{~nm}$, respectively with (i) showing the OA results and (ii) the CA results. In the OA measurement ( Figs. 3(a-i) and 3(b-i) ), we observe the typical reverse saturation absorption (RSA) $[16,18,29]$ at both wavelengths. Since the optical bandgap of $\mathrm{BiOBr}$ is much larger than the photo energy of incident lasers, we attribute the observed RSA to the two-photon absorption (TPA) at $800 \mathrm{~nm}$ and multi-photon absorption (MPA) at $1550 \mathrm{~nm}$. To extract the nonlinear coefficient $\beta$ of $\mathrm{BiOBr}$, we fit the measured OA data by $[19,36]$ :

$$
T_{\mathrm{OA}}(z) \simeq 1-\frac{1}{2 \sqrt{2}} \frac{\beta I_{0} L_{\mathrm{eff}}}{\left(1+\mathrm{x}^{2}\right)},
$$

where $T_{\mathrm{OA}}(z)$ is the normalized optical transmittance of $\mathrm{OA}$ measurement, and $x=\mathrm{z} / \mathrm{z}_{0}$, with $\mathrm{z}$ and $\mathrm{z}_{0}$ denoting the sample position relative to the focus and the Rayleigh length of the laser beam, respectively; $L_{\text {eff }}=\left(1-\mathrm{e}^{-\alpha_{0} L}\right) / \alpha_{0}$ is the effective sample thickness, with $\alpha_{0}$ and $L$ denoting the linear absorption coefficient and the sample thickness, respectively and $I_{0}$ is the irradiance intensity at focus. The $\beta$ 's at $800 \mathrm{~nm}$ (Fig. 3(a-i)) and $1550 \mathrm{~nm}$ (Fig. 3(b-i)) are measured to be $\sim 1.869 \times 10^{-7} \mathrm{~m} / \mathrm{W}$ and $\sim 1.554 \times 10^{-7} \mathrm{~m} / \mathrm{W}$, respectively. Such high nonlinear absorption $\beta$ is very favorable for high performance optical limiter applications.[8, 10]
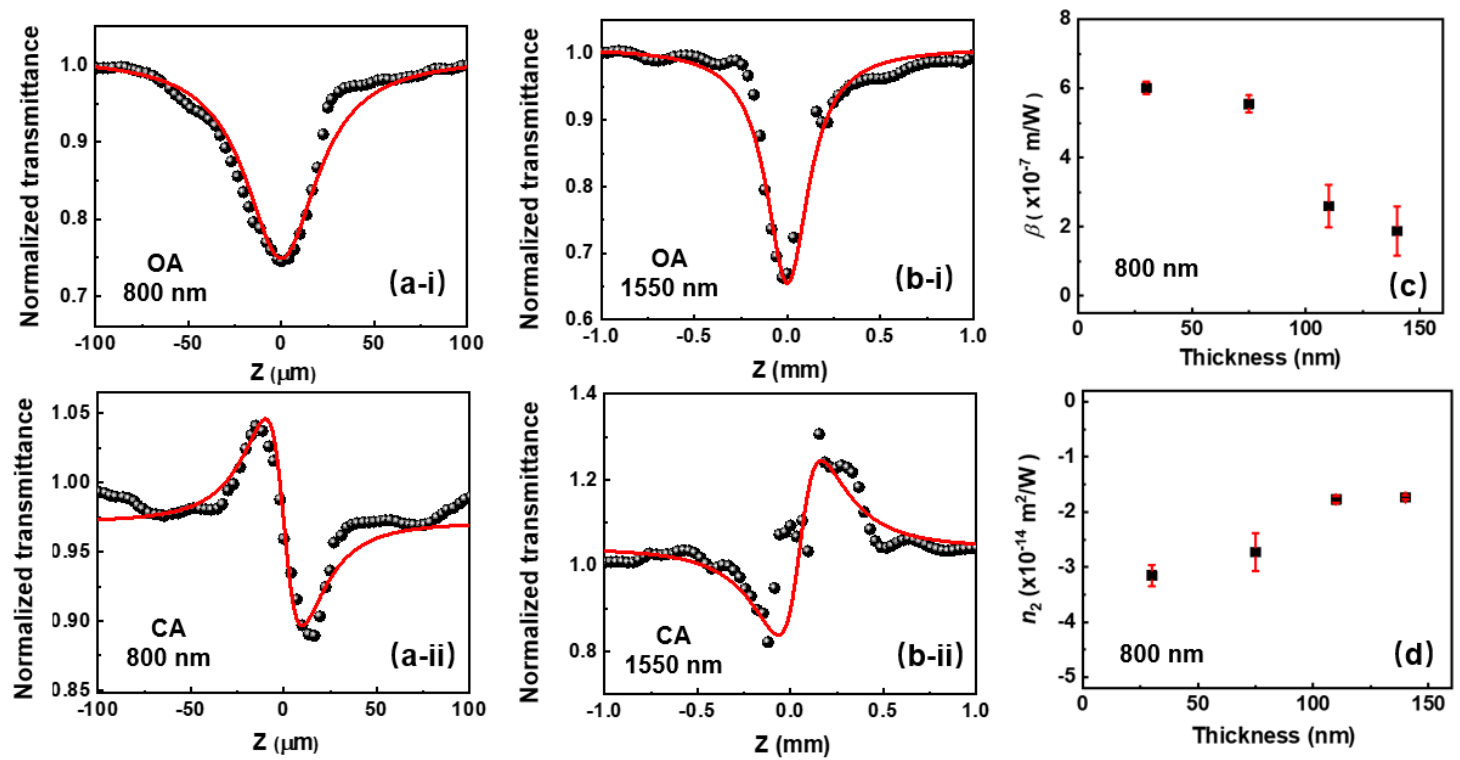

Fig. 3. (a) OA (i) and CA (ii) results at $800 \mathrm{~nm}$. (b) OA (i) and CA (ii) results at $1550 \mathrm{~nm}$. The thickness of the BiOBr sample is $\sim 140 \mathrm{~nm}$. (c) nonlinear absorption coefficient $\beta$ and (d) Kerr coefficient $n_{2}$ for BiOBr samples with different thicknesses.

We also investigate the nonlinear refraction of the BiOBr sample at both $800 \mathrm{~nm}$ and $1550 \mathrm{~nm}$ with the CA measurements, as shown in Figs. 3(a-ii) and (b-ii). In Fig. 3(a-ii), prominent peak-valley transmittance is observed for the CA measurement at $800 \mathrm{~nm}$, which corresponds to a negative Kerr coefficient $n_{2}$ and reflects optical selfdefocusing in $\mathrm{BiOBr}$ nanoflakes. In contrast, valley-peak transmittance is observed at $1550 \mathrm{~nm}$ in Fig. 3(b-ii), which is a reflection of optical self-focusing, resulting in a positive $n_{2}$. The $n_{2}$ value of $\mathrm{BiOBr}$ can be extracted from the measured CA results with [31]: 


$$
T_{\mathrm{CA}}\left(z, \Delta \Phi_{0}\right) \simeq 1+\frac{4 \Delta \Phi_{0} x}{\left(x^{2}+9\right)\left(x^{2}+1\right)},
$$

where $T_{\mathrm{CA}}\left(z, \Delta \Phi_{0}\right)$ is the normalized optical transmittance of CA measurement. $\Delta \Phi_{0}=2 \pi n_{2} I_{0} L_{\mathrm{eff}} / \lambda$ is the nonlinear phase shift, with $\lambda$ denoting the laser center wavelength. The measured Kerr coefficients $n_{2}$ 's at $800 \mathrm{~nm}$ and $1550 \mathrm{~nm}$ are $\sim-1.737 \times 10^{-14} \mathrm{~m}^{2} / \mathrm{W}$ and $\sim 3.824 \times 10^{-14} \mathrm{~m}^{2} / \mathrm{W}$, respectively. The transition from negative $n_{2}$ at $800 \mathrm{~nm}$ to positive $n_{2}$ at $1550 \mathrm{~nm}$ can be attributed to the dispersion of $n_{2}$ associated with the two-photon bandgap where $n_{2}$ is positive when the excitation photon energy (at $1550 \mathrm{~nm}$ ) is below the TPA bandedge (half-bandgap) while it becomes negative when the photon energy (at $800 \mathrm{~nm}$ ) is between the one-photo absorption and TPA edges [37, 38]. It should be note that the $n_{2}$ value of $\mathrm{BiOBr}$ is more than three orders of magnitude larger than that of $\mathrm{Si}[1]$, demonstrating its prominent Kerr nonlinearities and high potential for high-performance nonlinear photonic applications.

We further characterize the thickness dependent nonlinear response of BiOBr nanoflakes via $800 \mathrm{~nm} \mathrm{Z}$-scan measurements. The irradiance laser intensity was $0.202 \mathrm{GW} / \mathrm{cm}^{2}$. The measured $\beta$ and $n_{2}$ for BiOBr samples with different thicknesses are plotted in Figs. 3(c) and (d), respectively. It can be seen that $\beta$ and $n_{2}$ for the BiOBr nanoflakes is highly thickness dependent, where their absolute values increase when the sample thickness decreases from 140nm to $30 \mathrm{~nm}$. The dependence of nonlinear optical parameters on sample thickness is likely induced by localized defects in $\mathrm{BiOBr}$ nanoflakes, which would lead to more scattering and energy loss for thicker BiOBr nanoflakes, thus resulting in a decreased absolute values of $\beta$ and $n_{2}[36,39]$.

Table 1. Comparison of $\beta, n_{2}$, and figure of merit (FOM) of various 2D layered materials

\begin{tabular}{|c|c|c|c|c|c|c|}
\hline Material & Laser parameter & Thickness $(\mathbf{n m})$ & $\boldsymbol{\beta}(\mathbf{m} / \mathbf{W})$ & $\boldsymbol{n}_{\mathbf{2}}\left(\mathbf{m}^{\mathbf{2}} / \mathbf{W}\right)$ & FOM & Ref. \\
\hline Graphene & $1550 \mathrm{~nm}, 100 \mathrm{fs}$ & $5-7$ layers & $9 \times 10^{-8}$ & $-8 \times 10^{-14}$ & -0.574 & {$[9]$} \\
\hline $\mathrm{GO}$ & $800 \mathrm{~nm}, 100 \mathrm{fs}$ & $2 \times 10^{3}$ & $4 \times 10^{-7}$ & $1.25 \times 10^{-13}$ & 0.391 & {$[10]$} \\
\hline $\mathrm{MoS}_{2}$ & $1064 \mathrm{~nm}, 25 \mathrm{ps}$ & $2.5 \times 10^{4}$ & $-3.8 \times 10^{-11}$ & $1.88 \times 10^{-16}$ & -4.649 & {$[15]$} \\
\hline $\mathrm{WS}_{2}$ & $1040 \mathrm{~nm}, 340 \mathrm{fs}$ & 57.9 & $1.81 \times 10^{-8}$ & $-3.36 \times 10^{-16}$ & -0.018 & {$[16]$} \\
\hline $\mathrm{BP}$ & $1030 \mathrm{~nm}, 140 \mathrm{fs}$ & 15 & $5.845 \times 10^{-6}$ & $-1.635 \times 10^{-12}$ & -0.272 & {$[19]$} \\
\hline $\mathrm{BiOCl}$ & $800 \mathrm{~nm}, 100 \mathrm{fs}$ & $20-140$ & $4.25 \times 10^{-9}$ & $3.8 \times 10^{-15}$ & 1.118 & {$[30]$} \\
\hline $\mathrm{BiOBr}$ & $800 \mathrm{~nm}, 140 \mathrm{fs}$ & 30 & $6.011 \times 10^{-7}$ & $-3.155 \times 10^{-14}$ & -0.066 & This work \\
\hline $\mathrm{BiOBr}$ & $800 \mathrm{~nm}, 140 \mathrm{fs}$ & 140 & $1.869 \times 10^{-7}$ & $-1.737 \times 10^{-14}$ & -0.116 & This work \\
\hline $\mathrm{BiOBr}$ & $1550 \mathrm{~nm}, 140 \mathrm{fs}$ & 140 & $1.554 \times 10^{-7}$ & $3.824 \times 10^{-14}$ & 0.159 & This work \\
\hline
\end{tabular}

In Table 1 , we compare the measured $\beta$ and $n_{2}$ of $\mathrm{BiOBr}$ with other $2 \mathrm{D}$ layered materials. What is significant is that, $\mathrm{BiOBr}$ nanoflakes exhibit a large $\beta$ on the order of $10^{-7} \mathrm{~m} / \mathrm{W}$, which is much higher than many other layered materials, in particular being two orders of magnitude higher than $\mathrm{BiOCl}$. The Kerr coefficient $n_{2}$ is on the order of $10^{-14} \mathrm{~m}^{2} / \mathrm{W}$, which is close to that of graphene and GO, and is more than one order of magnitude higher than TMDCs and $\mathrm{BiOCl}$. These results confirm the superior nonlinear optical properties of $\mathrm{BiOBr}$ nanoflakes as an advanced nonlinear optical material.

\section{INTEGRATION ON SILICON PHOTONIC DEVICES}

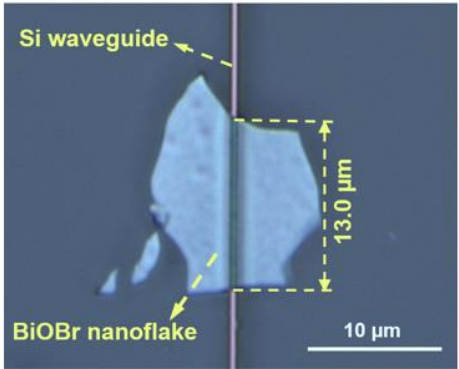

(a)

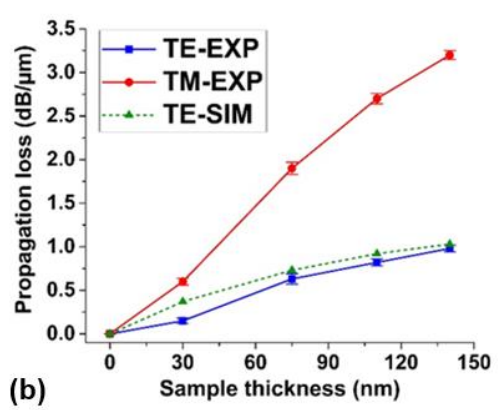

(b)

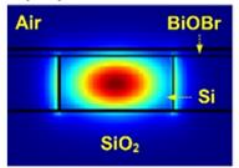

(c-i) $30 \mathrm{~nm} \mathrm{BiOBr}$

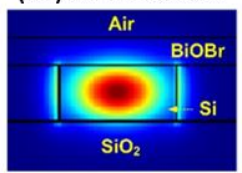

(c-iii) $110 \mathrm{~nm}$ BiOBr (c-iv) $140 \mathrm{~nm} \mathrm{BiOBr}$

Fig. 4 (a) Microscope image of a silicon integrated waveguides incorporated with BiOBr nanoflake. (b) Measured and simulated waveguide propagation loss of the hybrid waveguides for different $\mathrm{BiOBr}$ thicknesses. (c) TE (Ex) mode profile of the hybrid integrated waveguide for different BiOBr thicknesses: (i) $30 \mathrm{~nm}$, (ii) $75 \mathrm{~nm}$, (iii) $110 \mathrm{~nm}$, and (iv) $140 \mathrm{~nm}$.

Finally, we characterize the BiOBr nanoflakes integrated in 220-nm-thick silicon-on-insulator (SOI) waveguides [40] [1] on a 2- $\mu \mathrm{m}$-thick buried oxide (BOX) layer. BiOBr nanoflakes were transferred onto the silicon integrated 
waveguides using an all-dry transfer method [36, 41]. Fig. 4(a) shows a representative microscope image of a silicon integrated waveguide incorporated with $\mathrm{BiOBr}$ nanoflake. The width of the waveguide was $\sim 500 \mathrm{~nm}$. It can be seen that the $\mathrm{BiOBr}$ nanoflake is attached to the silicon integrated waveguide, with an overlap length of $\sim 13 \mu \mathrm{m}$. The thickness of the BiOBr nanoflake is $\sim 110 \mathrm{~nm}$.

The measured TE polarized insertion losses of the silicon waveguides with and without a $110-\mathrm{nm}-\mathrm{thick} \mathrm{BiOBr}$ nanoflake are $\sim 6.9 \mathrm{~dB}$ and $\sim 16.0 \mathrm{~dB}$, respectively. When we measured the insertion losses, the power of the input continuous-wave light was $\sim 0 \mathrm{dBm}$. The butt coupling loss was $\sim 3.0 \mathrm{~dB}$ each, or $\sim 6.0 \mathrm{~dB}$ for both. According to our previously fabricated devices, the waveguide propagation loss for single-mode silicon nanowire waveguides with a cross section of $500 \mathrm{~nm} \times 220 \mathrm{~nm}$ is about $\sim 3 \mathrm{~dB} / \mathrm{cm}[42,43]$, which is much lower than that of the hybrid waveguide. Therefore, the propagation loss of the hybrid waveguide can be given by:

$$
P L=\left(I L_{\text {hybrid }}-I L_{\text {silicon }}\right) / L_{\text {hybrid }},
$$

where $I L_{\text {hybrid }}$ and $I L_{\text {silicon }}$ are the insertion losses of the hybrid waveguide and the silicon waveguide without $\mathrm{BiOBr}$, respectively. $L_{\text {hybrid }}$ is the length of the $\mathrm{BiOBr}$ nanoflake on the silicon waveguide. The TE and TM polarized waveguide propagation losses extracted from the measured insertion losses is shown in Fig. 4(b). We measured the hybrid waveguides with four different BiOBr thicknesses. The data points depict the average values obtained from the experimental results of three samples and the error bars illustrate the variations for different samples. The propagation loss for TM polarization is much higher than that for TE polarization. Such difference is mainly caused by mode overlap and can be used for implementing polarizers [44]. We also perform mode analysis for the hybrid integrated waveguide using Lumerical FDTD commercial mode solving software. We used the in-plane (TE-polarized) $n$ and $k$ of 1- $\mu \mathrm{m}$-thick BiOBr obtained from the ellipsometry measurements (the values in Fig. 1(f) at $1550 \mathrm{~nm}$ ) in the FDTD simulation. Fig. 4(c) shows the TE mode profile of the hybrid integrated waveguides with different BiOBr thicknesses. For comparison, the simulated waveguide propagation losses are also shown in Fig. 4(b). It can be seen that the experimental propagation losses are close to the simulated propagation losses, which reflects the stability of the prepared $\mathrm{BiOBr}$ nanoflakes. We also note that the simulated propagation losses based on the $n$ and $k$ of $1-\mu \mathrm{m}$-thick $\mathrm{BiOBr}$ are slightly higher than the experimental propagation losses, with an increased difference between them for a decreased $\mathrm{BiOBr}$ thickness. This indicates that the intrinsic material loss actually increases with thickness, which could be attributed to any number of effects such as increased scattering loss and absorption induced by imperfect contact between the multiple layers as well as interactions between them.

\section{CONCLUSION}

In summary, we report on the nonlinear optical properties of $\mathrm{BiOBr}$ nanoflakes. A large nonlinear absorption coefficient $\beta$ on the order of $10^{-7} \mathrm{~m} / \mathrm{W}$ as well as a large Kerr coefficient $n_{2}$ on the order of $10^{-14} \mathrm{~m}^{2} / \mathrm{W}$ in $\mathrm{BiOBr}$ are observed in both $800 \mathrm{~nm}$ and $1550 \mathrm{~nm}$ Z-scan measurement. We also observe a strong dispersion in $n_{2}$, with $n_{2}$ changing sign from negative to positive when the wavelength is varied from $800 \mathrm{~nm}$ to $1550 \mathrm{~nm}$. The thicknessdependent nonlinear optical properties of $\mathrm{BiOBr}$ nanoflakes are demonstrated, where the magnitudes of $\beta$ and $n_{2}$ increase with decreasing thickness of the $\mathrm{BiOBr}$ nanoflakes. Finally, we integrate $\mathrm{BiOBr}$ nanoflakes into silicon integrated waveguides and measure their insertion loss, with the extracted waveguide propagation loss showing good agreement with mode simulations.

\section{ACKNOWLEDGEMENTS}

This work was supported by the Australian Research Council Discovery Projects Program (No. DP150102972 and DP190103186). We acknowledge Swinburne Nano Lab and Micro Nano Research Facility (MNRF) of RMIT University for the support in material characterization as well as Advanced Micro Foundry (AMF) Pte Ltd for the support in silicon device fabrication.

\section{REFERENCES}

[1] D. J. Moss, R. Morandotti, A. L. Gaeta, and M. Lipson, "New CMOS-compatible platforms based on silicon nitride and Hydex for nonlinear optics," Nature Photonics, vol. 7, no. 8, pp. 597-607, 2013.

[2] A. Pasquazi et al., "Micro-combs: A novel generation of optical sources," Physics Reports, vol. 729, pp. 1-81, 2018.

[3] J. Wu et al., "RF Photonics: An Optical Microcombs' Perspective," Ieee Journal Of Selected Topics In Quantum Electronics, vol. 24, no. 4, pp. 1-20, 2018.

[4] M. Z. Alam, I. De Leon, and R. W. Boyd, "Large optical nonlinearity of indium tin oxide in its epsilon-near-zero region," Science, vol. 352, no. 6287, pp. 795-7, May 132016.

[5] H. Bao et al., "Laser cavity-soliton microcombs," Nature Photonics, vol. 13, no. 6, pp. 384-389, 2019. 
[6] X. Xu et al., "Broadband RF Channelizer Based on an Integrated Optical Frequency Kerr Comb Source," Journal Of Lightwave Technology, vol. 36, no. 19, pp. 4519-4526, 2018.

[7] X. Xu, M. Tan, J. Wu, R. Morandotti, A. Mitchell, and D. J. Moss, "Microcomb-Based Photonic RF Signal Processing," IEEE Photonics Technology Letters, vol. 31, no. 23, pp. 1854-1857, 2019.

[8] G.-K. Lim et al., "Giant broadband nonlinear optical absorption response in dispersed graphene single sheets," Nature Photonics, vol. 5, no. 9, pp. 554-560, 2011.

[9] G. Demetriou et al., "Nonlinear optical properties of multilayer graphene in the infrared," Optics Express, vol. 24, no. 12, pp. 13033-43, Jun 132016.

[10] X. Zheng, B. Jia, X. Chen, and M. Gu, "In situ third-order non-linear responses during laser reduction of graphene oxide thin films towards on-chip non-linear photonic devices," Advanced Materials, vol. 26, no. 17, pp. 2699-703, May 2014.

[11] Y. Yang et al., "Invited Article: Enhanced four-wave mixing in waveguides integrated with graphene oxide," $A P L$ Photonics, vol. 3, no. 12, 2018.

[12] X. Xu et al., "Observation of Third-order Nonlinearities in Graphene Oxide Film at Telecommunication Wavelengths," Sci Rep, vol. 7, no. 1, p. 9646, Aug 292017.

[13] J. Wu et al., "Graphene Oxide Waveguide and Micro-Ring Resonator Polarizers," Laser \& Photonics Reviews, vol. 13, no. 9, 2019.

[14] Y. Yang et al., "Graphene-Based Multilayered Metamaterials with Phototunable Architecture for on-Chip Photonic Devices," ACS Photonics, vol. 6, no. 4, pp. 1033-1040, 2019.

[15] S. Bikorimana et al., "Nonlinear optical responses in two-dimensional transition metal dichalcogenide multilayer: WS2, WSe2, MoS2 and Mo0.5W0.5S2," Optics Express, vol. 24, no. 18, pp. 20685-95, Sep 52016.

[16] N. Dong et al., "Dispersion of nonlinear refractive index in layered WS2 and WSe2 semiconductor films induced by twophoton absorption," Optics Letters, vol. 41, no. 17, pp. 3936-9, Sep 12016.

[17] H. Liu, Y. Li, Y. S. You, S. Ghimire, T. F. Heinz, and D. A. Reis, "High-harmonic generation from an atomically thin semiconductor," Nature Physics, vol. 13, no. 3, pp. 262-265, 2016.

[18] X. Zheng et al., "Characterization of nonlinear properties of black phosphorus nanoplatelets with femtosecond pulsed Zscan measurements," Optics Letters, vol. 40, no. 15, pp. 3480-3, Aug 12015.

[19] I. A. Tieshan Yang, Han Lin, Yang Bao, Sherman Jun Rong Tan, Scott Fraser, Kian Ping Loh, and Baohua Jia, , "Anisotropic third-order nonlinearity in pristine and lithium hydride intercalated black phosphorus," ACS Photonics, vol. 5, p. 8, 2018.

[20] T. Yang et al., "Anisotropic Third-Order Nonlinearity in Pristine and Lithium Hydride Intercalated Black Phosphorus," ACS Photonics, vol. 5, no. 12, pp. 4969-4977, 2018.

[21] F. Xia, H. Wang, D. Xiao, M. Dubey, and A. Ramasubramaniam, "Two-dimensional material nanophotonics," Nature Photonics, vol. 8, no. 12, pp. 899-907, 2014.

[22] X. Liu, Q. Guo, and J. Qiu, "Emerging Low-Dimensional Materials for Nonlinear Optics and Ultrafast Photonics," Advanced Materials, vol. 29, no. 14, Apr 2017.

[23] A. Autere, H. Jussila, Y. Dai, Y. Wang, H. Lipsanen, and Z. Sun, "Nonlinear Optics with 2D Layered Materials," Advanced Materials, vol. 30, no. 24, p. e1705963, Jun 2018.

[24] J. Di, J. Xia, H. Li, S. Guo, and S. Dai, "Bismuth oxyhalide layered materials for energy and environmental applications," Nano Energy, vol. 41, pp. 172-192, 2017.

[25] M. Guan et al., "Vacancy associates promoting solar-driven photocatalytic activity of ultrathin bismuth oxychloride nanosheets," Journal Of The American Chemical Society, vol. 135, no. 28, pp. 10411-7, Jul 172013.

[26] H. Feng et al., "Modulation of Photocatalytic Properties by Strain in 2D BiOBr Nanosheets," ACS Appl Mater Interfaces, vol. 7, no. 50, pp. 27592-6, Dec 232015.

[27] D. Cui et al., "Band-gap engineering of $\mathrm{BiOCl}$ with oxygen vacancies for efficient photooxidation properties under visible-light irradiation," Journal of Materials Chemistry A, vol. 6, no. 5, pp. 2193-2199, 2018.

[28] H. Yu et al., "Liquid-Phase Exfoliation into Monolayered BiOBr Nanosheets for Photocatalytic Oxidation and Reduction," ACS Sustainable Chemistry \& Engineering, vol. 5, no. 11, pp. 10499-10508, 2017.

[29] H. Li et al., "Facet-dependent nonlinear optical properties of bismuth oxychloride single-crystal nanosheets," Journal of Materials Chemistry C, vol. 6, no. 32, pp. 8709-8716, 2018.

[30] R. Chen, X. Zheng, Y. Zhang, Y. Tang, and T. Jiang, "Z-scan measurement of nonlinear optical properties of BiOCl nanosheets," Appl Opt, vol. 54, no. 21, pp. 6592-7, Jul 202015.

[31] A. A. S. Mansoor Sheik-Bahae, Tai-Huei Wei, David J. Hagan and E. W . Van Stryland, "Sensitive Measurement of Optical Nonlinearities Using a Single Beam," IEEE Journal of Quantum Electronics, vol. 26, no. 4, p. 10, 1990.

[32] J. E. D. Davies, "Solid state vibrational spectroscopy - III [1] the infrared and Raman spectra of the bismuth (III) oxide halides," J. Inorg, Nucl. Chem., vol. 35, no. 5, p. 4, 1973.

[33] H. Feng et al., "Construction of 2D lateral pseudoheterostructures by strain engineering," 2D Materials, vol. 4, no. 2, 2017.

[34] Y. C. Chang et al., "Realization of mid-infrared graphene hyperbolic metamaterials," Nat Commun, vol. 7, p. 10568, Feb 42016.

[35] L. Jia et al., "Highly nonlinear BiOBr nanoflakes for hybrid integrated photonics," APL Photonics, vol. 4, no. 9, 2019. 
[36] P. Li et al., "Two-Dimensional CH3NH3PbI3 Perovskite Nanosheets for Ultrafast Pulsed Fiber Lasers," ACS Appl Mater Interfaces, vol. 9, no. 14, pp. 12759-12765, Apr 122017.

[37] M. S.-B. A. A. Said, D. J. Hagan, T. H. Wei, J. Wang, J. Young, and E. W Van Stryland,, "Determination of boundelectronic and free-carrier nonlinearities in ZnSe, GaAs, CdTe, and ZnTe," Journal Of The Optical Society Of America B-optical Physics, vol. 9, no. 3, p. 10, 1992.

[38] M. Sheik-Bahae, D. J. Hagan, and E. W. Van Stryland, "Dispersion and band-gap scaling of the electronic Kerr effect in solids associated with two-photon absorption," Physical Review Letters, vol. 65, no. 1, pp. 96-99, Jul 21990.

[39] J. Zhang, T. Jiang, X. Zheng, C. Shen, and X. Cheng, "Thickness-dependent nonlinear optical properties of CsPbBr3 perovskite nanosheets," Optics Letters, vol. 42, no. 17, pp. 3371-3374, Sep 12017.

[40] W. Bogaerts and L. Chrostowski, "Silicon Photonics Circuit Design: Methods, Tools and Challenges," Laser \& Photonics Reviews, vol. 12, no. 4, p. 1700237, 2018.

[41] M. B. Andres Castellanos-Gomez, Herre S. J. van der Zant and Gary A. Steele, "Deterministic transfer of twodimensional materials by all-dry viscoelastic stamping," 2D Materials, 2013.

[42] J. Wu, T. Moein, X. Xu, and D. J. Moss, "Advanced photonic filters based on cascaded Sagnac loop reflector resonators in silicon-on-insulator nanowires," APL Photonics, vol. 3, no. 4, 2018.

[43] J. Wu et al., "Nested Configuration of Silicon Microring Resonator With Multiple Coupling Regimes," IEEE Photonics Technology Letters, vol. 25, no. 6, pp. 580-583, 2013.

[44] W. H. Lim et al., "Graphene oxide-based waveguide polariser: from thin film to quasi-bulk," Optics Express, vol. 22 , no. 9, pp. 11090-8, May 52014. 